\title{
PRODUCCIÓN LOCAL DE OBJETOS CERÁMICOS DURANTE LOS PERÍODOS TARDÍO E INCA EN EL VALLE DE TAFÍ (TUCUMÁN, ARGENTINA)
}

\author{
LOCAL POTTERY PRODUCTION POTTERY DURING LATE AND INCA \\ PERIODS IN THE TAFÍ VALLEY (TUCUMÁN, ARGENTINA)
}

\author{
María Cecilia Páez ${ }^{1,2}$ y Rita Plá
}

\begin{abstract}
Uno de los aspectos destacados en el análisis de la dinámica de las sociedades prehispánicas es su capacidad para producir los bienes que consumen, en este caso, la cerámica que utilizarán en sus actividades cotidianas, sean estas de índole doméstica o simbólico/ritual. Arqueológicamente, las primeras etapas de una investigación que se oriente en tal sentido apuntan a determinar la presencia de potenciales fuentes de arcilla, recurso primario para la manufactura cerámica, y su afinidad composicional con los fragmentos arqueológicos.

Presentamos los resultados de los Análisis por Activación Neutrónica (AAN) realizados sobre 54 muestras procedentes del valle de Tafí. De ellas, 50 corresponden a fragmentos arqueológicos asignados a los períodos Tardío e Inca dentro de la cronología regional y cuatro a muestras de arcillas de distintos puntos del valle. La afinidad en la composición multielemental de parte de la muestra orienta las interpretaciones hacia una manufactura de carácter local, atendiendo los recaudos interpretativos propios de un conjunto numéricamente reducido.
\end{abstract}

Palabras claves: análisis por Activación Neutrónica, producción cerámica, períodos Tardío e Inca.

A central issue in analyzing the dynamics of pre-Hispanic societies is their ability to produce the goods they consume, in this case, the pottery they used in their daily activities be they domestic or symbolic/ritual nature. One of the first stages of a program of archaeology study focused on such issues u involves identifying potential clay sources, a primary resource in ceramic manufacturing, and its compositional affinity with archaeological fragments.

In this paper we present the results of a Neutron Activation Analysis (NAA) conducted on 54 samples from the Tafi Valley in northwestern Argentina. Of these, 50 are archaeological fragments assigned to the Late and Inca Periods within the local regional chronology, while four are clay samples from across the valley. The multi-elemental affinity of sample compositions supports an interpretation of local pottery manufacturing, although the conclusions are tempered by the small sample in this initial study.

Key words: Neutron Activation Analysis, pottery production, Late and Inca Periods.

La disponibilidad de recursos minerales potencialmente utilizables en la manufactura cerámica en el valle de Tafí plantea varias interrogantes en torno a su uso efectivo en la producción de las vasijas que hoy se observan en el registro arqueológico. La posibilidad de manufacturar cerámica en los propios centros poblados en donde luego se dan las instancias de uso tiene importantes implicancias para la dinámica de esa sociedad. En primer lugar, ya sea que se trate de una actividad a nivel de la unidad doméstica o en la forma de una práctica especializada y controlada, supone cierta independencia de otros espacios que pueden ser geográficamente cercanos o no. Esto conlleva particularidades en la estructura interna de la sociedad, en este caso, de Tafí, como en los vínculos fuera del valle. Las relaciones laborales, la organización de la producción, los lazos entre quienes proveen alfarería y quienes la "consumen", la intervención en forma y grado del Estado en la manera en que se produce y los destinos de los productos son solo algunas de las instancias que estructuran una sociedad en función de una manufactura local o no local de los bienes usados. En este marco, los análisis de procedencia

1 CONICET, Consejo Nacional de Investigaciones Científicas y Técnicas. Av. Rivadavia 1917 (C1033AAJ), Buenos Aires, Argentina.

2 División Arqueología, Museo de Ciencias Naturales, Edificio Anexo, Unidades de Investigación, Universidad Nacional de La Plata, Av. 122 y 60 (1900), La Plata, Argentina. ceciliapaez@ conicet.gov.ar

3 Departamento Química Nuclear, Gerencia de Química Nuclear y Ciencias de la Salud, GAATEN. Comisión Nacional de Energía Atómica, Av. del Libertador 8250, (1429) Buenos Aires, Argentina. rpla@cae.cnea.gov.ar 
constituyen el primer paso para el abordaje de estas cuestiones, para lo cual fue necesario evaluar en primera instancia, la disponibilidad de fuentes de arcilla.

En trabajos de campos sucesivos en 2005 y 2006 se pudieron identificar ocho sectores con depósitos de arcilla, que fueron luego analizados en el laboratorio a fin de determinar su aptitud para la manufactura (Cuenya et al. 2007; Páez 2010; Páez y Manasse 2013). Estudios acerca de las características geomecánicas y mineralógicas de estas materias primas permitieron determinar que la mayor parte de los depósitos muestreados podrían haber sido utilizados en la producción de cerámica en tiempos prehispánicos, obteniendo un cuadro distribucional de arcillas que cubre distintas localizaciones del valle, algunas muy próximas a contextos arqueológicos del período Tardío e Inca (Cuenya et al. 2007; Páez 2010).

No obstante, la determinación de su efectiva utilización en la manufactura solo es posible mediante la correlación entre la composición química (elementos traza) de las arcillas muestreadas y los fragmentos procedentes de los sitios arqueológicos. El Análisis por Activación Neutrónica (AAN) constituye una de las técnicas analíticas más convenientes para los estudios de procedencia cerámica, ampliamente utilizada en las investigaciones arqueológicas desde la década de 1980 (Bishop 1980; Bishop et. al. 1982; Bishop y Neff 1989; Tite 1999). Opera sobre las concentraciones de una gran variedad de elementos químicos, y a base de su asociación es posible inferir comportamientos de producción.

En este trabajo se presentan los resultados de los análisis de AAN realizados sobre una muestra de fragmentos y arcillas cocidas procedentes del valle de Tafí, a partir de los cuales se analizará la procedencia del registro cerámico de momentos Tardío e Inca.

\section{El Valle de Tafí}

El valle de Tafí conforma una cuenca intermontana emplazada a una altura ente 1.850 y $2.500 \mathrm{msm}$ en el borde oriental de la región Valliserrana correspondiente a la provincia de Tucumán, entre los paralelos $26^{\circ} 45^{\prime}$ y $26^{\circ} 58^{\prime}$ de latitud sur y los meridianos de $65^{\circ} 37^{\prime}$ y $65^{\circ} 52^{\prime}$ de longitud oeste (Figura 1). Geomorfológicamente constituye una depresión tectónica que separa dos sistemas montañosos importantes: las cumbres
Calchaquíes, al norte y las sierras de Aconquija, al sur. Las cumbres de Mala Mala lo delimitan por el este, apartándolo de la llanura oriental, junto al cerro Ñuñorco. Hacia el oeste, el cerro Muñoz lo separa del valle de Santa María, dibujándose un paso natural a través de El Infiernillo (Arenas et al. 2007).

Las investigaciones que se vienen desarrollando en el área desde hace varias décadas sugieren una profundidad ocupacional del valle de al menos 2.500 años (Manasse 2001, 2007, 2012). Las sociedades del último milenio, recorte temporal de esta investigación, han estado marcadamente relacionadas con la dinámica valliserrana. El período Tardío (900 AD-1.480 AD) se caracteriza por una arquitectura de estructuras rectangulares deprimidas conocidas como "casas-pozo" (González y Núñez Regueiro 1960). La alfarería típica para estos momentos es conocida como Santa María (o Santamariana), definida a partir de piezas de forma cerrada (urnas) y abierta (pucos), con decoración en dos colores (negro sobre blanco) o tres (negro y rojo / blanco), diseños geométricos y figurativos, principalmente con representación antropomorfa de la cara (Perrota y Podestá 1978; Weber 1978). Otro estilo cerámico frecuente durante este período es el Famabalasto Negro Grabado, que consiste en varias morfologías con superficie de color negro pulido, y diseños de líneas incisas. La alfarería tosca, de superficies alisadas o peinadas, habría sido empleada en las actividades domésticas, y aún funerarias.

El valle de Tafí constituye uno de los dominios más orientales del Tawantinsuyu. Aunque las referencias arquitectónicas son escasas, la abundancia de alfarería asignada a los estilos Inca Provincial e Inca Mixto (sensu Calderari y Williams 1991) da cuenta de la incorporación del Valle a la dinámica imperial. La alfarería Santa María del período precedente siguió siendo preponderante en los conjuntos, además de aquella que se define como Fase Inca que, en caso de Tafí, estaría representada por los estilos Famabalasto Negro sobre Rojo, Yocavil Polícromo y Belén.

La información que aquí presentaremos procede de dos contextos arqueológicos que corresponden, uno al período de Desarrollos Regionales y otro al período Inca (Figura 1). El primero -S.Tuc.Tav. 15se asigna a un cementerio Santamariano que habría contenido aproximadamente cuarenta entierros en urnas. Se encuentra localizado en el noroeste del valle, en la zona de La Ovejería, a 26 51' 19,3” de 


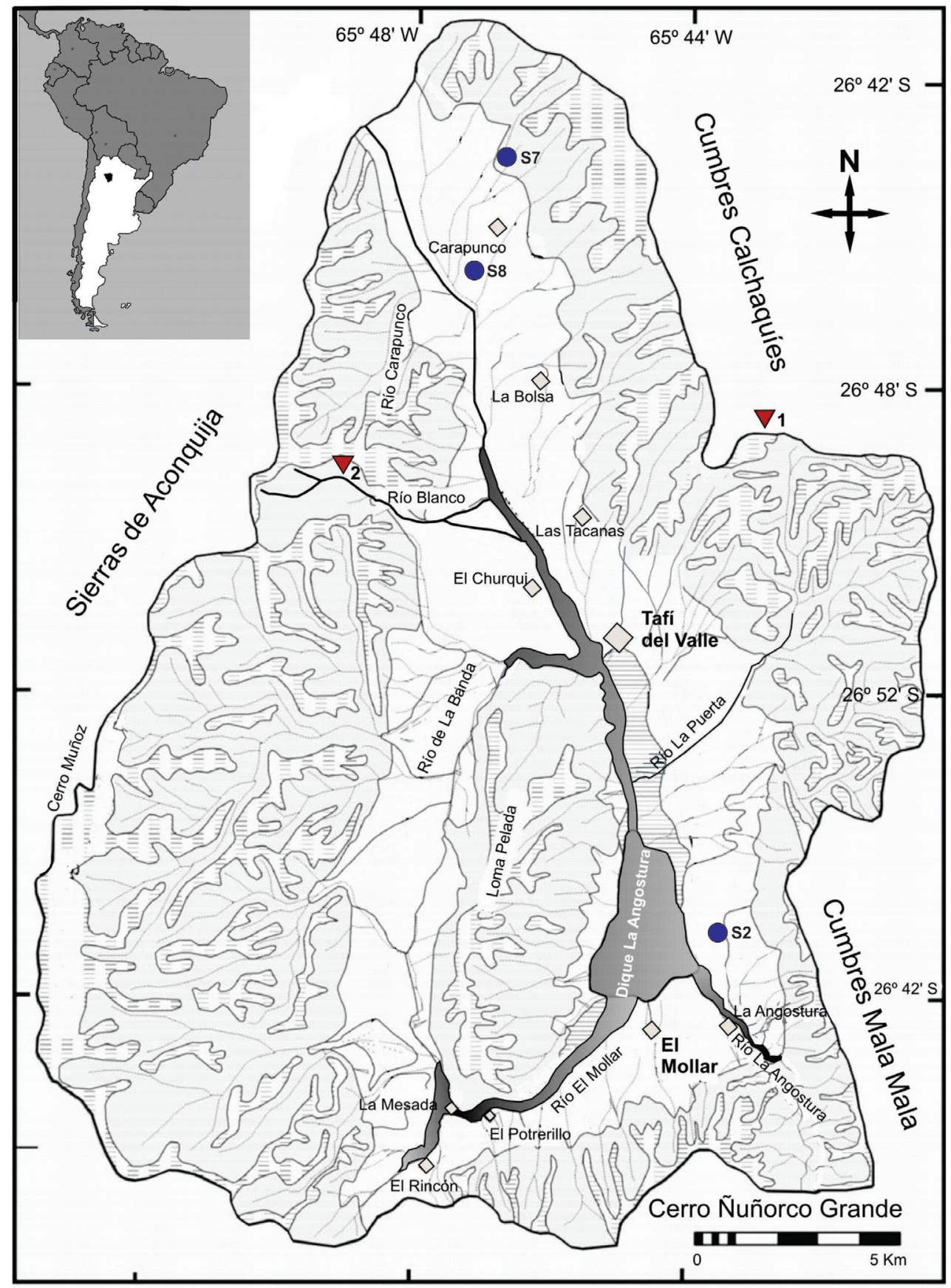

1. Sitio LCZVIIIS1

2. Sitio S.Tuc.Tav.15

\section{S2, S7 y S8. Fuentes de arcilla}

Figura 1. Mapa del valle de Tafí (Tucumán, Argentina). En la imagen se indica la ubicación de los contextos arqueológicos de los que proceden los fragmentos cerámicos y de las fuentes de arcilla analizadas, y su relación espacial con los sitios. S2: Loma Bola; S7: Perfil La Bolsa 1 y 2; S8; El Pinar.

Map of the Tafí valley (Tucumán, Argentina). The map shows the location of the archaeological contexts where sherds were obtained and the distribution of clays sources in relation to sites (or with respect to the sites). S2: Loma Bola; S7: La Bolsa 1 and 2; S8: El Pinar. 
latitud sur, y $65^{\circ} 45^{\prime} 00,0$ " de longitud oeste (Leiva Benegas 2003). Comprende un espacio de $90 \mathrm{~m}$ en sentido E-O y $100 \mathrm{~m} \mathrm{~N}$-S, lo que supone una variación altimétrica importante. El segundo-LCZVIIIS1- está localizado a $26^{\circ} 50^{\prime} 00^{\prime \prime}$ de latitud sur y $65^{\circ} 41^{\prime} 30^{\prime}$ " de longitud oeste, en un promontorio que domina visualmente el valle en el faldeo meridional de las cumbres Calchaquíes (Manasse 2002). Se trata de un conjunto conglomerado de estructuras circulares, subcirculares, cuadrangulares y subcuadrangulares, con un abundante registro cerámico propio del período Inca (Páez 2010; Patané Aráoz 2008).

\section{Análisis por Activación Neutrónica (AAN)}

El conjunto analizado comprende 54 muestras $(\mathrm{n}=54)$ que incluyen 50 fragmentos cerámicos y cuatro muestras de arcillas locales. Del total de fragmentos, 20 corresponden al contexto tardío $(n=20)$, en tanto los 30 restantes se asignan al contexto incaico $(n=30)$. Se pretendió abarcar toda la diversidad estilística propia de cada período cronológico considerado, aunque se priorizó la representatividad cuantitativa de los estilos cerámicos Santa María e Inca, que son los más representativos de cada momento. La Tabla 1 reúne las características de procedencia, asignación estilística y morfológica de los fragmentos que integran la muestra de análisis, y la gráfica de algunos estilos se aprecia en la Figura 2.

Es importante tener en cuenta que si bien la muestra es reducida, constituye un punto de partida a partir del cual explorar aspectos que tienen que ver con la producción local/no local de estilos que se reconocen como propios de la región y aun de Tafí, como es el caso del estilo Santamariano en sus distintas variedades. De la misma manera, surgen interrogantes frente a la presencia de materiales propios de regiones más alejadas. Tal es el caso de aquellos Famabalasto Negro sobre Rojo o Yocavil, sobre los cuales se asume que estarían vinculados a movimientos poblacionales propiciados por el Estado como parte de una estrategia de control poblacional (Lorandi 1983, 1984, 1991; Lorandi et al. 1991; Williams 1996; Williams y Lorandi 1986). La importante representatividad de este último en el contexto LCZVIIIS1, alerta acerca de su relevancia en el marco de la política incaica en Tafí.

Desde un punto de vista comparativo, la vinculación o desvinculación entre los materiales asociados al tardío local y al momento incaico, nos permite profundizar en aquellos aspectos sociales y políticos que se ocultan tras las continuidades o discontinuidades en el uso, por ejemplo, de las fuentes de arcilla. Por esta razón, y frente a la necesidad de vincular la muestra fragmentaria con un espacio físico concreto, se decidieron incorporar muestras de arcilla locales que hubieran sido analizadas en laboratorio y respecto de las cuales se tuviera certeza de su aptitud para la manufactura cerámica. La inconveniencia de la comparación entre arcillas crudas y arcillas cocidas (fragmentos arqueológicos) hizo necesaria la confección de briquetas experimentales que reprodujeran lo más cercano posible las características de la cerámica analizada. En este sentido, fue necesario controlar los aspectos vinculados con la cocción -atmósfera y temperatura-como requisito fundamental en orden a reproducir las características alcanzadas en la cerámica prehispánica. A tal fin se seleccionaron cuatro muestras de arcilla procedentes de los siguientes puntos de muestreo: (1) El Pinar (muestra (EP-VdT), (2) Loma Bola (muestra LB-VdT), (3) y (4) Perfil La Bolsa -donde se utilizaron dos niveles de paleosuelo (muestras PBL1-VdT y PLB2-VdT respectivamente). La selección se realizó sobre una base de ocho puntos de muestreo (Páez 2010; Páez y Manasse 2013), a partir de aquellos depósitos que asumían las condiciones geomecánicas más óptimas para su uso en la producción cerámica de acuerdo con los análisis de laboratorio (Cuenya et al. 2007).

La pasta se preparó sin adición de antiplástico, utilizando las inclusiones naturales de la arcilla. Inicialmente se agregó agua al sedimento para su hidratación, mediante la cual alcanzaría el grado de plasticidad necesario. Luego se colocó la preparación en una superficie de yeso a fin de que eliminara el agua sobrante y se procedió al amasado y preparación de las briquetas, de dimensiones $6 \mathrm{~cm}$ x $6 \mathrm{~cm} \times 1$ $\mathrm{cm}$ (Figura 3). Estas se dejaron secar a temperatura ambiente y posteriormente se cocieron en horno eléctrico a temperatura de $850{ }^{\circ} \mathrm{C}$, de acuerdo con la información de cocción obtenida para los fragmentos arqueológicos (Páez 2005).

Los análisis de AAN en los fragmentos arqueológicos y las arcillas cocidas fueron realizados por el Laboratorio de Técnicas Analíticas Nucleares del Centro Atómico Ezeiza (Buenos Aires, Argentina). Se molieron muestras de aproximadamente $100 \mathrm{mg}$ usando un mortero de ágata, que luego se secaron en estufa durante $24 \mathrm{~h}$ a una temperatura de $100^{\circ} \mathrm{C}$. Las irradiaciones se realizaron en el reactor RA-3 (flujo térmico $\left.3.10^{13} \mathrm{~cm}^{-2} \cdot \mathrm{s}^{-1} ; 4,5 \mathrm{Mw}\right)$, determinándose 
Tabla 1. Características de procedencia y asignación estilística y morfológica de los fragmentos cerámicos que integran la muestra analizada; $\mathrm{n}=50$.

Provenience, style and shape classification for sherds analized by NAA; $n=50$.

\begin{tabular}{|c|c|c|c|}
\hline Muestra & Procedencia & Estilo cerámico & Forma de la pieza \\
\hline EL10 & S.Tuc.Tav. 15 & Santa María N/B1. & Cerrada \\
\hline EL200 & S.Tuc.Tav. 15 & Santa María N/Bl. & Indeterminada \\
\hline EL193 & S.Tuc.Tav. 15 & Santa María N/B1. & Indeterminada \\
\hline EL245 & S.Tuc.Tav. 15 & Tosco & Cerrada \\
\hline EL343 & S.Tuc.Tav. 15 & Santa María N/B1. & Indeterminada \\
\hline EL367 & S.Tuc.Tav. 15 & Santa María N/Bl. & Indeterminada \\
\hline EL44 & S.Tuc.Tav. 15 & Santa María N/B1. & Cerrada \\
\hline EL33 & S.Tuc.Tav. 15 & Santa María N/Bl. & Cerrada \\
\hline EL690 & S.Tuc.Tav. 15 & Santa María NyR/Bl. & Cerrada \\
\hline EL441 & S.Tuc.Tav. 15 & Tosco & Cerrada \\
\hline EL187 & S.Tuc.Tav. 15 & Santa María N/B1. & Cerrada \\
\hline EL396 & S.Tuc.Tav. 15 & Tosco & Cerrada \\
\hline EL36 & S.Tuc.Tav. 15 & Santa María N/B1. & Cerrada \\
\hline EL20 & S.Tuc.Tav. 15 & Santa María N/Bl. & Cerrada \\
\hline EL684 & S.Tuc.Tav. 15 & Santa María N/B1. & Cerrada \\
\hline EL357 & S.Tuc.Tav. 15 & Santa María N/B1. & Indeterminado \\
\hline EL687 & S.Tuc.Tav. 15 & Santa María NyR/B1. & Cerrada \\
\hline EL57 & S.Tuc.Tav. 15 & Santa María N/B1. & Indeterminado \\
\hline EL119 & S.Tuc.Tav. 15 & Santa María N/B1. & Cerrada \\
\hline LC60e11 & LCZVIIIS1 & Santa María NyR/Bl. & Cerrada \\
\hline LC58c11 & LCZVIIIS1 & Santa María N/B1. & Cerrada \\
\hline LC59a13 & LCZVIIIS1 & Famabalasto NG & Abierta \\
\hline LC58a7 & LCZVIIIS1 & Inca & Abierta \\
\hline $\mathrm{LC} 58 \mathrm{c} 2$ & LCZVIIIS1 & Inca & Abierta \\
\hline LC58a10 & LCZVIIIS1 & Santa María N/B1. & Abierta \\
\hline LC58c15 & LCZVIIIS1 & Inca & Abierta \\
\hline LC59a10 & LCZVIIIS1 & Inca & Cerrada \\
\hline LC58c22 & LCZVIIIS1 & Santa María N/B1. & Abierta \\
\hline LC59c25 & LCZVIIIS1 & Tosco & Cerrada \\
\hline LC57c35 & LCZVIIIS1 & Inca & Abierta \\
\hline LC58c3 & LCZVIIIS1 & Santa María N/B1. & Abierta \\
\hline LC59c36 & LCZVIIIS1 & Yocavil & Abierta \\
\hline LC57c11 & LCZVIIIS1 & Famabalasto N/R & Abierta \\
\hline LC59c22 & LCZVIIIS1 & Tosco & Cerrada \\
\hline LC61b48 & LCZVIIIS1 & Santa María N/R & Cerrada \\
\hline LC61b43 & LCZVIIIS1 & Tosco & Cerrada \\
\hline LC61b4 & LCZVIIIS1 & Inca & Abierta \\
\hline LC61b15 & LCZVIIIS1 & Famabalasto NG & Abierta \\
\hline LC59c15 & LCZVIIIS1 & Santa María N/B1. & Abierta \\
\hline Lc60a7 & LCZVIIIS1 & Famabalasto N/R & Abierta \\
\hline $\mathrm{LC} 58 \mathrm{a} 2$ & LCZVIIIS1 & Tosco & Cerrada \\
\hline LC57g35 & LCZVIIIS1 & Famabalasto N/R & Abierta \\
\hline LC57c39 & LCZVIIIS1 & Famabalasto N/R & Abierta \\
\hline LC58a15 & LCZVIIIS1 & Inca & Abierta \\
\hline LC61a2 & LCZVIIIS1 & Inca & Cerrada \\
\hline LC57g11 & LCZVIIIS1 & Inca & Abierta \\
\hline LC59c39 & LCZVIIIS1 & Famabalasto N/R & Abierta \\
\hline LC61b11 & LCZVIIIS1 & Yocavil & Abierta \\
\hline LC60c16 & LCZVIIIS1 & Inca & Abierta \\
\hline
\end{tabular}




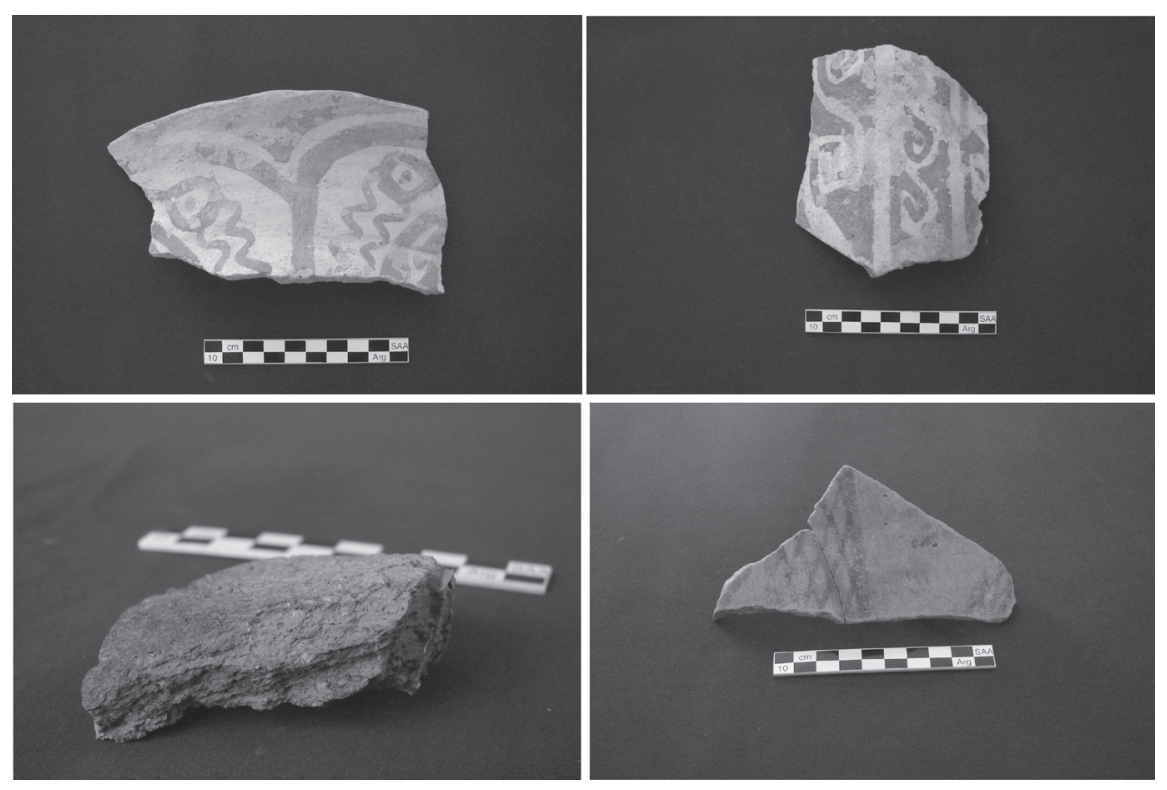

Figura 2. Fragmentos cerámicos analizados por AAN; $\mathrm{n}=50$.

Ceramic fragments analyzed by $N A A ; n=50$.

Referencias: Sup. Izq. y Der. estilo cerámico Santa María negro sobre blanco; Inf. Izq. estilo cerámico tosco; Inf. Der. Estilo cerámico Santa María negro sobre rojo.

References: Upper left and right: Santa María black on white; lower left: utilitarian; lower right: Santa María black on red.

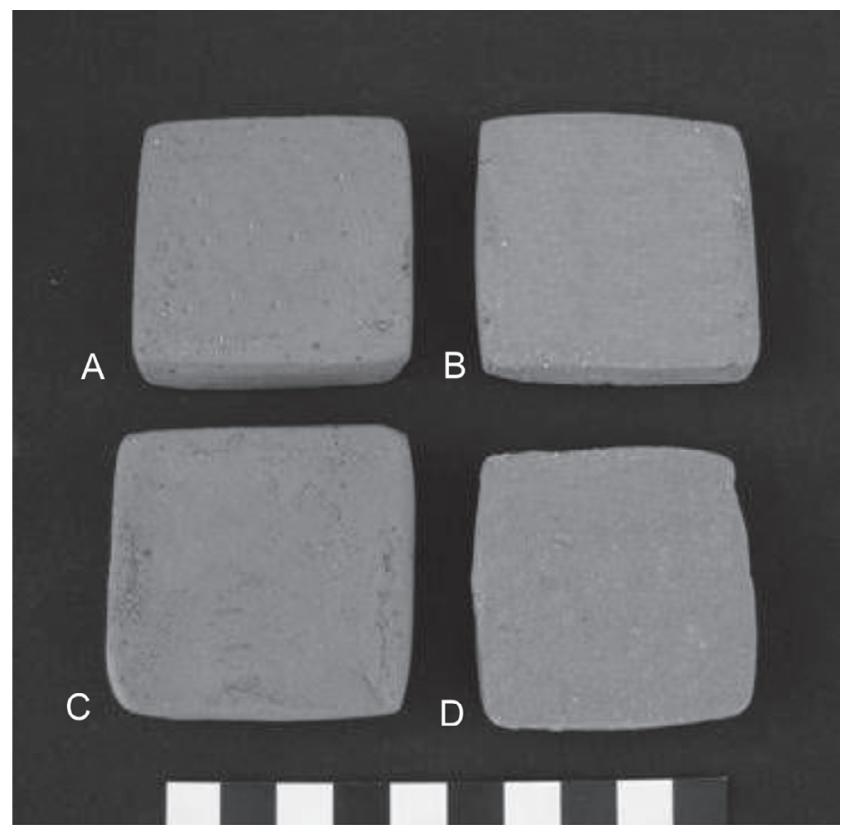

Figura 3. Briquetas experimentales elaboradas a partir de las cuatro fuentes de arcilla muestreadas. A. El Pinar (muestra EP-VdT), C. Loma Bola (muestra LB-VdT), B y D. Perfil La Bolsa (muestras PBL1-VdT y PLB2-VdT, respectivamente).

Experimental briquettes made with four clay samples from the Tafi valley. A. El Pinar (sample EP-VdT), C. Loma Bola (sample LB-VdT), B and D. Perfil La Bolsa (samples PBL1-VdT and PLB2-VdT respectively). 
20 elementos químicos, que incluyen: $\mathrm{As}, \mathrm{Ba}, \mathrm{Ce}$, $\mathrm{Co}, \mathrm{Cr}, \mathrm{Cs}, \mathrm{Eu}, \mathrm{Fe}, \mathrm{Gd}, \mathrm{Hf}, \mathrm{La}, \mathrm{Lu}, \mathrm{Rb}, \mathrm{Sb}, \mathrm{Sc}$, $\mathrm{Sm}, \mathrm{Tb}, \mathrm{Th}, \mathrm{U}$ y Yb. Los datos composicionales de los fragmentos y las arcillas fueron tratados estadísticamente en conjunto.

\section{Resultados de los Análisis}

Obtenidos los resultados de la composición química de la muestra total se realizó el análisis estadístico de la información utilizando 19 de los 20 elementos determinados ${ }^{1}$, distribuidos en tierras raras (Ce, Eu, La, Lu, Sm, Yb), alcalinos $(\mathrm{Ba}, \mathrm{Cs}, \mathrm{Rb}$ ), metales de transición (Sc, Hf, Co, $\mathrm{Cr}, \mathrm{Fe}, \mathrm{Tb}$ ), actínidos (Th, U), y metaloides ( $\mathrm{Sb}$ ). Inicialmente se aplicó estadística descriptiva que permitió evaluar el comportamiento de la muestra vinculado a las magnitudes de las concentraciones elementales. La Tabla 2 presenta los valores de media, desviación estándar y rango para las muestras agrupadas de acuerdo con su asignación estilística. Se pudo observar una importante similitud en el comportamiento químico de los grupos Famablasto Negro sobre Rojo y Yocavil, que son los que más se asemejan del conjunto. Los fragmentos Famabalasto Negro Grabado no se parecen a los homónimos Negro sobre Rojo; por el contrario, se acercan más al comportamiento de las pastas Santa María. Este agrupamiento conserva la identidad de cada uno de los tres subtipos -Negro sobre blanco, Negro sobre Rojo y Negro y Rojo sobre Blanco-. Los fragmentos incaicos, al contrario de lo que podría esperarse, se acercan más al perfil de las pastas Santa María que de aquellas Famabalasto y Yocavil. Finalmente, la mayor parte de las pastas de la cerámica tosca o no decorada presenta un perfil multielemental más cercano a aquellas Famabalasto y Yocavil, que a las Santa María e Inca.

El análisis numérico multivariado -Análisis de Componentes Principales- se realizó con el software estadístico SPSS 15.0 con transformación logarítmica de los datos, cálculo de matriz de correlación y rotación Varimax. De esta manera se obtuvieron cinco componentes que explican el $76,6 \%$ de la variación total. El primer componente $(30,4 \%)$ tiene alta contribución de metales de transición, Sc, Fe, $\mathrm{Co}, \mathrm{Cr}$; un metaloide (Sb), un actínido (U) y los alcalinos $\mathrm{Cs}$ y $\mathrm{Rb}$. El segundo componente $(23,3 \%)$ tiene aporte importante de Th (un actínido que suele asociarse a las tierras raras); tierras raras: $\mathrm{La}, \mathrm{Sm}$, Hf y Ce (TR livianas), Eu (TR mediana) y Lu, Tb e $\mathrm{Yb}$ (TR pesadas). Si bien las contribuciones de $\mathrm{Hf}$ y Sm están algo repartidas entre los dos primeros componentes (Hf) y el segundo y tercero ( $\mathrm{Sm})$, su mayor carga es para el segundo componente. Los siguientes tres componentes tienen aporte importante de un solo elemento: Ba (alcalino térreo) en el tercero, As (metaloide) en el cuarto y Ta (metal de transición) en el quinto componente.

Respecto de los resultados obtenidos se efectuó un análisis de conglomerados jerárquicos, utilizando el método de vinculación promedio Ward y la distancia euclideana al cuadrado, a fin de identificar grupos con perfil químico similar, que más tarde fueron ajustados mediante el análisis discriminante utilizando la distancia Mahalanobis (Ratto et al. 2007). Se definieron en principio tres grupos predictivos, a base de la semejanza de sus perfiles químicos, que luego fueron ajustados a dos.

A partir del análisis discriminante se pudieron asociar las arcillas a uno de los grupos predictivos (GP1) que reúne además fragmentos de ambos contextos, en tanto el otro grupo (GP2) quedó conformado solo por fragmentos. La Figura 4 muestra la distribución de los fragmentos y fuentes de arcilla en el espacio factorial indicando los dos grupos conformados. La Tabla 3 representa la composición de estos agrupamientos en relación con los estilos cerámicos de cada contexto arqueológico.

De acuerdo con esto, en primer lugar se observa una asociación de las arcillas muestreadas con fragmentos de ambos contextos. Para el caso de S.Tuc.Tav. 15 (período Tardío), se asocian a materiales de estilo cerámico Santa María Negro sobre Blanco, Santa María Negro y Rojo sobre Blanco y no decorados o toscos. Para LCZVIIIS1 (período Inca), la asociación química se daría con la mayor parte de los fragmentos Famabalasto Negro sobre Rojo, todos los fragmentos Yocavil y no decorados, y un fragmento de estilo Inca.

Es preciso considerar que la distancia entre el contexto tardío y la fuente Loma Bola es de aproximadamente $10 \mathrm{~km}$ en línea recta $-15 \mathrm{~km}$ pensando que hay que subir y bajar el cerro Pelado-, en tanto para el caso de El Pinar y ambos perfiles de La Bolsa el trayecto se reduce aproximadamente a la mitad. En el análisis espacial hay que tener presente, no obstante, que el cementerio podría haber albergado piezas de lugares distantes del valle, que pudieran estar cercanas a la localización de la fuente de arcilla. De esta manera, la distancia actual entre el lugar de entierro y la fuente de materia 


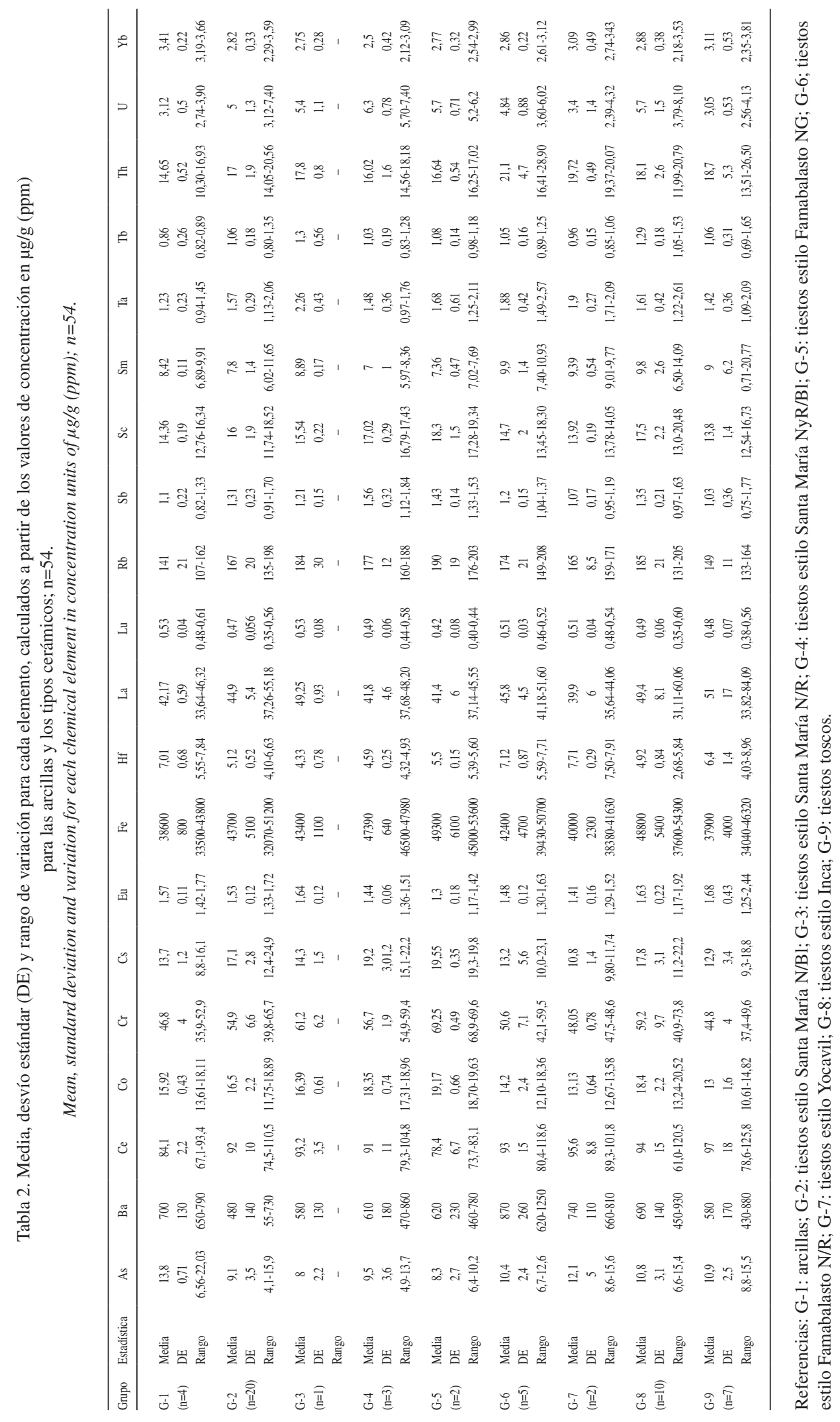




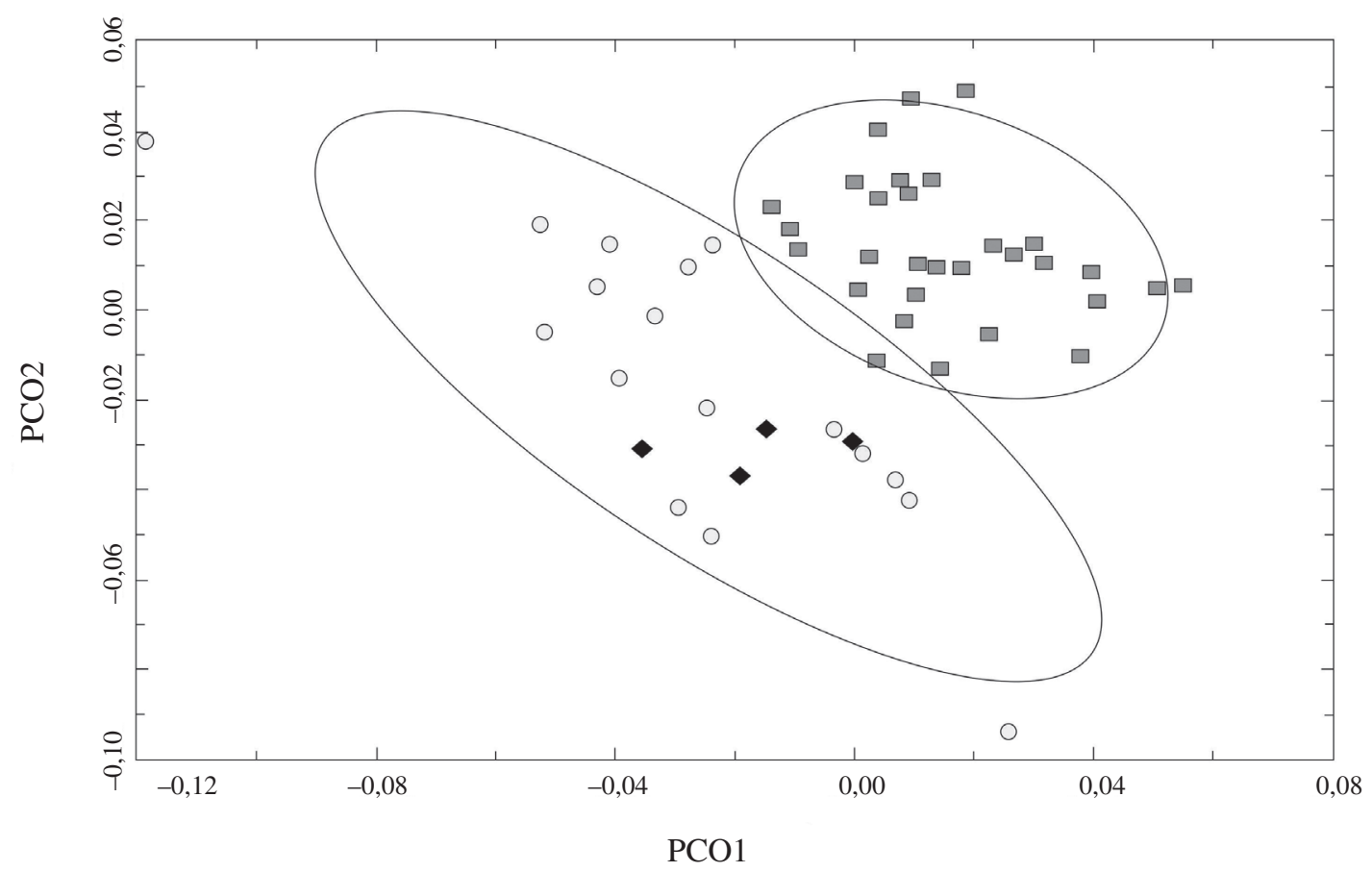

Figura 4. Agrupamiento de los fragmentos cerámicos y muestras de arcilla en el espacio factorial definido por los factores 1 y $2 ; \mathrm{n}=54$. Grouping of sherds and clay samples in the factorial space defined by factors 1 and $2 ; n=54$.

Tabla 3. Relación entre los agrupamientos de fragmentos (GP 1 y 2) y los estilos cerámicos para cada contexto arqueológico; $\mathrm{n}=50$.

Relationship between sherd groups GP 1 and 2 and ceramic styles for each archaeological context; $n=50$.

\begin{tabular}{cccccccccccc}
\hline & \multicolumn{1}{c}{ S.TUC.TAV. 15 $(\mathrm{n}=20)$} & \multicolumn{1}{c}{ LCZIIIS1 $(\mathrm{n}=30)$} \\
\hline GP & G-2 & G-4 & G-9 & G-2 & G-3 & G-4 & G-5 & G-6 & G-7 & G-8 & G-9 \\
\hline 1 & 5 & 0 & 2 & 0 & 0 & 0 & 0 & 4 & 2 & 1 & 2 \\
2 & 9 & 1 & 1 & 5 & 1 & 1 & 2 & 1 & 0 & 9 & 0 \\
S/GP & 1 & 1 & 0 & 0 & 0 & 0 & 0 & 0 & 0 & 0 & 2 \\
\hline Total & 15 & 2 & 3 & 5 & 1 & 1 & 2 & 5 & 2 & 10 & 4 \\
\hline
\end{tabular}

Referencias: G-2: tiestos estilo Santa María N/B1; G-3: tiestos estilo Santa María N/R; G-4: tiestos estilo Santa María NyR/B1; G-5: tiestos estilo Famabalasto NG; G-6; tiestos estilo Famabalasto N/R; G-7: tiestos estilo Yocavil; G-8: tiestos estilo Inca; G-9: tiestos toscos.

prima solo podría ser una medida aproximada de la vinculación espacial entre uno y otro. Para el caso del contexto incaico, dos de las fuentes se encuentran cercanas, El Pinar a 3,5 km y el Perfil La Bolsa, a 2,5 km, en tanto la restante se ubica a más de $10 \mathrm{~km}$ del sitio (Figura 1).

Es también remarcable que la totalidad de los fragmentos Santa María (en sus distintas variantes estilíticas) e Inca del contexto incaico presentan el mismo perfil químico (GP2), que difiere de las pastas Famabalasto, Yocavil y toscas que se agrupan en el otro grupo predictivo (GP1). Para el caso de las pastas Santa María del contexto Tardío, parte se asocia al GP1 y parte lo hace con el GP2.

Finalmente, algunas muestras caen fuera de las elipses de confianza. Dos de ellas, que corresponden al contexto tardío, están muy cerca de la elipse que delimita el GP 2. Las otras dos -que pertenecen 
al incaico-, están fuera de las elipses y alejadas, principalmente la primera. En este sentido, el análisis de nuevos tiestos y arcillas puede contribuir a mejorar la interpretación de los agrupamientos hallados, lo que parece ser necesario, también, a los fines de resolver el mínimo solapamiento de las dos elipses en el que queda involucrada una muestra correspondiente a un fragmento tosco del contexto S.Tuc.Tav. 15.

\section{Discusión y Conclusiones}

Los datos hasta aquí obtenidos generan certezas del carácter local de la producción prehispánica durante el Segundo Milenio de la Era Cristiana, al tiempo que nos alertan del uso de recursos accesibles en el espacio geográfico no tan cercano como es el caso de la fuente Loma Bola. Esta información química presentada se corresponde, asimismo, con datos obtenidos desde otras vías analíticas que indican una correlación entre el análisis mineralógico de las arcillas (Cuenya et al. 2007) y el estudio petrográfico de la muestra fragmentaria (Páez 2010). A partir de ellos se detectó la presencia de vidrio volcánico en densidades muy bajas en la composición mineral de algunas pastas. El análisis de la forma, el tamaño, y el redondeamiento de los granos permitió sostener que estas inclusiones estarían contenidas en las arcillas que se usaron para la manufactura, lo que encuentra un ajuste conveniente con los resultados del análisis mineralógico realizado por Difracción de Rayos $\mathrm{X}$ en las muestras de arcillas. Esta característica está presente en los dos contextos cerámicos que aquí analizamos -S.Tuc.Tav.15 y LCZVIIIS1-.

La conformación de dos grupos predictivos a partir del análisis por AAN -el primero de ellos asociado a las cuatro fuentes de arcilla-, sugiere un distanciamiento entre los conjuntos cerámicos. El GP1 concentra los estilos Famabalasto Negro sobre Rojo y Yocavil Polícromo, ambos asociados a poblaciones trasplantadas como parte de políticas estatales procedentes del oeste catamarqueño. Los datos químicos indicarían que piezas de estos estilos habrían sido manufacturadas dentro del valle con una técnica totalmente diferente de la que se observa para la cerámica de estilo Santa
María. Estas observaciones serían congruentes con los movimientos poblacionales a los que se asocia el estilo cerámico fuera de su región de origen, descartando que hayan sido piezas elaboradas fuera del valle y transportadas allí para su uso. A este conjunto aparece asociado el perfil multielemental de los fragmentos toscos y un único caso incaico.

El GP2 incluye la mayor parte de los materiales Santa María (de ambos contextos), los Famabalasto Negro Grabado, un solo fragmento Famabalasto Negro sobre Rojo y la mayor parte de los que corresponden al estilo Inca. Es interesante, en este agrupamiento, la relación entre la mayor parte de los materiales santamarianos del contexto tardío y sus homónimos y los incaicos del contexto Inca, lo que podría sugerir una continuidad en el tiempo de algunos patrones de producción cerámica. En este sentido, los estudios mineralógicos realizados sobre muestras de ambos sitios indican que los cambios en la tecnología de producción entre los períodos Tardío e Inca en Tafí fueron puntuales y evidentes solo en una fracción de las piezas cerámicas, particularmente aquellas vinculadas a los espacios de legitimación del Estado (Páez 2011; Páez y Sardi 2014). Por otro lado, la desvinculación entre el GP2 y las fuentes arcillosas incorporadas al análisis se presenta como un punto de partida importante sobre el que focalizar en futuros trabajos. La ampliación de la muestra debido a la incorporación de nuevas fuentes de arcilla y mayor cantidad de fragmentos cerámicos contribuirá a una mayor claridad en los agrupamientos establecidos y, por ende, a un mejor conocimiento de características de la producción alfarera en el valle de Tafí.

Agradecimientos: Deseamos expresar nuestro agradecimiento a María Eugenia Prieto y Nicolás Scivetti por su colaboración con las imágenes, a Rodrigo Invernizzi por su ayuda con el tratamiento estadístico de los datos y a los revisores anónimos, cuyos comentarios permitieron mejorar la versión inicial del manuscrito. Los AAN fueron realizados en el marco de la Beca Doctoral de la primera autora, con fondos del Consejo Nacional de Investigaciones Científicas y Técnicas. La responsabilidad de lo expresado es exclusiva de las autoras. 


\section{Referencias Citadas}

Arenas, P., B. Manasse y E. Noli 2007. Paisajes y procesos sociales en Tafí del Valle. En Paisajes y Procesos Sociales en Tafi del Valle, compilado por P. Arenas, B. Manasse y E. Noli, pp. 13-44. Imprenta de la Universidad Nacional de Tucumán, San Miguel de Tucumán.

Bishop, R. 1980. Aspects of ceramic compositional modelling. En Models and Methods in Regional Exchange, editado por R.E. Fry, pp. 47-65. SAA Paper $\mathrm{N}^{\circ}$ 1.Society for American Archaeology, Washington D.C.

Bishop, R.L., R.L. Rands y G.R. Holley 1982. Ceramic compositional analysis in archaeological perspective. En Advances in Archaeological Method and Theory 5, editado por M.B. Schiffer, pp. 275-330. Academic Press, New York.

Bishop, R.L. y H. Neff 1989. Compositional data analysis in archaeology. En Archaeological Chemistry IV, editado por R. Allen, pp. 57-85. American Chemical Society, Washington D.C.

Calderari, M. y V. Williams 1991. Reevaluación de los estilos cerámicos incaicos en el Noroeste Argentino. Comechingonia 9:75-95.

Cuenya, P, R. Ovejero, B. Manasse y M.C. Páez 2007. Materias primas para cerámica ¿probables fuentes? Ponencia presentada en VIII Jornadas de Comunicaciones de la Facultad de Ciencias Naturales e Instituto Miguel Lillo, Universidad Nacional de Tucumán, Tucumán.

González, A.R. y V. Núñez Regueiro 1960. Preliminary report in archaeological research in Tafí del Valle, NW Argentina. Akten des $34^{\circ}$ Internationalen Amerikanisten Kongress, pp. 18-25. Viena.

Leiva Benegas, S.J. 2003. Analysis of Possible Pre-Selection Patterns Regarding to Cementeries in Calchaqui Valley, Tucumán, Argentine. Tesis de licenciatura inédita, Göteborgs Universitet Institutionen för arkeologi C-uppsats, Gotemburgo.

Lorandi, A.M. 1983. Olleros del Inka en Catamarca, Argentina. Gaceta Arqueológica Andina 2:6-10.

Lorandi, A.M. 1984. Soñocamayoc. Los olleros del Inka en los centros manufactureros del Tucumán. Revista del Museo de La Plata, Nueva Serie 8:303-327.

Lorandi, A.M. 1991. Evidencias en torno a los mitmaqkuna incaicos en el NOA. Antropológica 9:212-236.

Lorandi, A.M., B. Cremonte y V. Williams 1991. Identificación étnica de los mitmakuna en el establecimiento inka de PotreroChaquiago. Actas del XI Congreso Nacional de Arqueología Chilena Tomo II, pp. 195-200.

Manasse, B. 2002. Una historia alternativa sobre el pasado prehispánico del valle de Tafí, Producciones Científicas NOA 2002. SEDECyT, Universidad Nacional de Catamarca. Publicación en CD.

Manasse, B. 2007. Tiempo antes de la conquista española en el Valle de Tafí. En Paisajes y Procesos Sociales en Tafí del Valle, compilado por P. Arenas, B. Manasse y E. Noli, pp. 135-163. Imprenta de la Universidad Nacional de Tucumán, San Miguel de Tucumán.

Manasse, B. 2012. Arqueología en el Borde Andino del Noroeste Argentino. Sociedades del Último Milenio en el Valle de Tafí,
Prov. de Tucumán, República Argentina. Tesis de doctorado inédita. Facultad de Ciencias Naturales y Museo, Universidad Nacional de La Plata, La Plata.

Páez, M.C. 2005. El Tardío en el Valle de Tafí, Prov. de Tucumán: Estudio de Material Alfarero Santamariano. Tesis de licenciatura inédita. Escuela de Arqueología, Universidad Nacional de Catamarca, Catamarca.

Páez, M.C. 2010. Tecnología Alfarera del Último Milenio de Ocupación Aborígen del Valle de Tafí (Prov. de Tucumán). Tesis de doctorado inédita. Facultad de Ciencias Naturales y Museo, Universidad Nacional de La Plata, La Plata.

Páez, M.C. 2011. De presencias y ausencias. Cambios y continuidades en la tecnología alfarera de las sociedades del valle de Tafí. En La Cerámica en la Materialización de la Sociedad: Transformaciones, Metáforas y Reproducción Social, editado por M.C. Páez y G. De La Fuente, pp. 79-89. British Archaeological Report (BAR), Oxford.

Páez, M.C. y B. Manasse 2013. Materias primas arcillosas en el valle de Tafí (Tucumán, Argentina). Evaluación de su disponibilidad y aptitud para la manufactura cerámica prehispánica. Actas del XI Congreso y Exposición Internacional de la Industria Cerámica, del Vidrio, Refractarios y Suministros, pp. 333-338. Universidad Nacional del Centro de la Provincia de Buenos Aires, Olavarría.

Páez, M.C. y M. Sardi 2014. Changes in pottery production under Inka rule in a frontier site (Tafí Valley, Argentina): a mineralogical análisis. Ñawpa Pacha: Journal of Andean Archaeology 34:61-78.

Patané Aráoz, C.J. 2008. Arqueología de los Encuentros: Lo Inka y lo Local en un sitio en las montañas de un valle del NOA. Estudiando al Pukara de las Lomas Verdes (Tafi del Valle, Prov. de Tucumán). Tesis de licenciatura inédita. Escuela de arqueología, Universidad Nacional de Catamarca, Catamarca.

Perrota, E y C. Podestá 1978. Contribution to the San José and Santa María Cultures, Northwest Argentina. En Advances in Andean Archaeology, editado por D. Browman, pp. 525-551. Mouton-The Hague, París-Chicago.

Ratto, N., A. Feely y R. Plá 2007. La Producción Alfarera en el bolsón de Fiambalá (Departamento Tinogasta, Catamarca) y su Alcance Extra-Regional. En Cerámicas Arqueológicas: Perspectivas Arqueométricas para su Análisis e Interpretación, editado por N. Ratto y B. Cremonte, pp. 123-145. Editorial de la Universidad Nacional de Jujuy, San Salvador de Jujuy.

Tite, M. 1999. Pottery production, distribution, and consumption. The contribution of the physical sciences. Journal of Archaeological Method and Theory 6:181-233.

Weber, R. 1978. A seriation of the late prehistoric Santa Maria culture of Northwestern. Fieldiana Anthropology 68:49-98.

Williams, V. 1996. Arqueología inca en la Región Central de Catamarca (República Argentina). Tesis de doctorado inédita. Facultad de Ciencias Naturales y Museo, Universidad Nacional de La Plata, La Plata.

Williams, V. y A.M. Lorandi 1986. Evidencias funcionales de un establecimiento incaico en el noroeste argentino. Comechingonia 4:135-148. 


\section{Nota}

1 Para As, en la muestra EL119, el valor faltante se reemplazó por la media de la distribución para poder trabajar con una matriz completa. Se eliminó Gd de la matriz de datos, debido al número de valores por debajo del límite de detección o con alto valor de incertidumbre. 